\title{
L'enseignement supérieur et le Processus de Bologne en Slovaquie
}

\section{Beata Kosová et Gilles Rouet}

\section{(2) OpenEdition \\ 1 Journals}

Édition électronique

URL : https://journals.openedition.org/ries/3075

DOI : $10.4000 /$ ries.3075

ISSN : 2261-4265

Éditeur

France Education international

\section{Édition imprimée}

Date de publication : 1 avril 2013

Pagination : 19-25

ISBN : 978-2-85420-599-2

ISSN : $1254-4590$

Référence électronique

Beata Kosová et Gilles Rouet, "L'enseignement supérieur et le Processus de Bologne en Slovaquie », Revue internationale d'éducation de Sèvres [En ligne], 62 I avril 2013, mis en ligne le 01 avril 2016, consulté le 06 juillet 2021. URL : http://journals.openedition.org/ries/3075 ; DOI : https://doi.org/ $10.4000 /$ ries.3075

Ce document a été généré automatiquement le 6 juillet 2021.

(c) Tous droits réservés 


\title{
L'enseignement supérieur et le Processus de Bologne en Slovaquie
}

\author{
Beata Kosová et Gilles Rouet
}

1 La Slovaquie comptait, fin 2012, vingt universités publiques, trois établissements d'État ${ }^{1}$, douze établissements d'enseignement supérieur privés et quatre filiales d'établissements étrangers. Le système d'enseignement supérieur a connu une expansion rapide après 1989 . Ainsi, le nombre d'étudiants de premier cycle est passé de 60000 à plus de 200000 entre 1989 et 2009 (30\% d'étudiants en régime d'études externes, $58 \%$ de femmes et $2 \%$ d'étrangers). Les effectifs baissent pour la troisième année consécutive ( $-4 \%$ entre 2009 et 2010), une évolution qui n'est pas seulement imputable à la démographie du pays, mais aussi à l'évolution du système. Les établissements d'enseignement supérieur emploient plus de 21000 personnes (en équivalent temps plein), dont un peu plus de $46 \%$ d'enseignants et $7 \%$ de chercheurs. On dénombre moins de 10000 enseignants dans le supérieur, de statuts différents : professeur, docent (maître de conférences habilité), assistant, lecteur et professeur invité. Le personnel est sous contrat à durée déterminée (pour cinq ans généralement), renouvelable (comme dans la plupart des services publics slovaques).

2 Le nombre de professeurs est un critère crucial en Slovaquie, car chaque programme d'études doit avoir un " garant ", un enseignant-chercheur ayant le grade de professeur des universités. Cette disposition est problématique pour la garantie de certains programmes existants ou pour de nouvelles créations, en particulier dans des champs nouveaux pour lesquels des dispositifs d'habilitation de professeurs n'ont pas été mis en place.

3 Une commission d'accréditation indépendante a été créée en 1990, avec pour missions le contrôle et l'évaluation de l'enseignement et de la recherche des établissements slovaques. Chaque programme et chaque établissement doivent faire l'objet d'une accréditation tous les six ans. La Commission formule également des propositions en ce qui concerne la création, la fusion, l'annulation, la séparation et le changement de nom des universités publiques ou privées ainsi que de leurs facultés. L'excellence en recherche, le nombre et la qualité du personnel enseignant et l'infrastructure 
technique sont les principaux facteurs de l'évaluation générale, qui conditionne le type de reconnaissance des établissements : dix-huit ont été reconnus comme universités fin 2012.

4 La loi autorise le statut d'établissement d'enseignement supérieur privé depuis 1996, sous réserve d'obtenir une accréditation officielle. Depuis cette loi, le nombre de ce type d'établissements n'a cessé d'augmenter et leur effectif d'étudiants est en augmentation constante (sans représenter plus de $10 \%$ du nombre total d'étudiants), ce qui s'explique en grande partie par le type de programmes proposés (économie, droit) et la demande dans ces programmes (en particulier en droit: de nombreux étudiants slovaques tentent leur admission en République tchèque, le nombre d'admis dans l'enseignement public slovaque étant limité).

5 Malgré une multiplication par trois du nombre d'étudiants de premier cycle et de doctorat, la part des dépenses publiques consacrées à l'enseignement supérieur dans le PIB est passée de $0,98 \%$ à $0,71 \%$ entre 1989 et 2010. Cette diminution des dépenses réelles a évidemment affecté la qualité du système d'enseignement supérieur public.

6 Les établissements, autogérés et autonomes, doivent tenter de maximiser leur dotation budgétaire qui se décompose en deux parties : le financement institutionnel (transfert du budget de l'État et des subventions du fonds national de la recherche) qui s'appuie sur des critères relatifs, en particulier, à l'activité scientifique et pédagogique des équipes, et le financement par projets après mise en concurrence (subventions de programmes spécifiques, fonds structurels, etc.).

7 Le budget alloué par l'État par dotation comprend l'ensemble des frais liés au fonctionnement des établissements, mais aussi les bourses destinées aux étudiants. En 2003, une loi a permis le transfert de la propriété et de la gestion du patrimoine immobilier de l'État vers les universités, renforçant donc leur autonomie; un amendement, en janvier 2009, a autorisé les universités à engager leurs biens, sous certaines conditions, pour financer des entreprises conjointes de recherche avec des entreprises privées, ou encore pour louer leurs bâtiments à des fins commerciales.

8 L'entrée à l'université se fait généralement sur concours ouvert aux titulaires d'un certificat de fin d'études secondaires (maturita). Des procédures d'admission particulières sont appliquées individuellement aux candidats d'origine étrangère. Les formes du concours de recrutement varient d'une université et d'une faculté à l'autre. Les études supérieures sont gratuites dans les universités publiques, à l'exception de certains frais administratifs (frais de procédures d'admission ou de délivrance de diplôme). Un système de bourses d'études est garanti par l'État sur des critères d'excellence ou sociaux.

9 Des études courtes professionnalisantes sont proposées au sein d'établissements différents des universités : les écoles professionnelles (odbornej školy), académies de commerce, écoles spécialisées. Les programmes de ces filières courtes ont souvent été élaborés en contact étroit avec des institutions étrangères d'enseignement professionnel, de manière à correspondre en particulier aux critères des marchés de l'Union européenne. Les études durent généralement trois ans et sont sanctionnées à l'issue par un examen (absolutorium). De nombreux étudiants de certains de ces établissements (en particulier de tourisme) cherchent à poursuivre leurs études au sein des universités. 
10 La réforme du processus d'accréditation a conduit à une diminution du nombre de programmes d'enseignement proposés et une nouvelle politique de frais d'inscription a été instaurée en 2009. Ainsi, bien que les études dans les universités publiques soient initialement gratuites, elles peuvent devenir payantes lorsque l'étudiant doit recommencer une année universitaire, par exemple à la suite d'un échec aux examens. Dans ce cas, le montant de l'inscription peut avoisiner le millier d'euros, ce qui peut poser un problème aux familles aux revenus modestes. Cependant, ces cas sont assez rares, car la pédagogie du système tout entier est plutôt fondée sur un accompagnement après une sélection initiale. La question des frais de scolarité concerne plutôt les étudiants qui choisissent un régime externe d'études et bénéficient alors d'aménagements importants en termes d'heures de cours et d'obligations. Globalement, plus d'un tiers des étudiants cumule les études avec une autre activité, en général un emploi.

11 Le domaine des «sciences sociales et services» regroupe plus de la moitié de la population étudiante. Il s'agit d'un ensemble assez hétérogène comprenant la philosophie, les sciences politiques, le droit, l'histoire, la psychologie, l'économie et les formations pour les métiers de l'enseignement. On note en particulier l'importance des effectifs dans ces deux dernières filières.

12 Entre 2000 et 2010, le nombre d'étudiants européens effectuant des études en Slovaquie a augmenté de 1560 à 8 179, ce qui reste faible par rapport au nombre total d'étudiants. Cette augmentation est encore plus importante pour les doctorants : de 113 à 942. Mais les étudiants tchèques représentant la moitié des étudiants étrangers, il faut relativiser ce résultat. La stratégie volontariste de certaines universités pour le développement de programmes d'études en langues étrangères est fondamentale pour augmenter le nombre d'étudiants étrangers. En effet, le déséquilibre est manifeste : la Slovaquie est classée parmi les pays ayant les taux les plus élevés d'émigration étudiante. D'après Eurostat, près de $14 \%$ des étudiants slovaques poursuivent des études à l'étranger (2009) et le nombre de doctorants slovaques dans les pays de l'Union européenne est passé de 366 en 2004 à 1548 en 2009. Mais il faut noter que la République tchèque est la destination de $70 \%$ des étudiants slovaques faisant des études à l'étranger.

\section{Bilan de l'implantation du Processus de Bologne}

13 La mise en œuvre des principes de la Déclaration de Bologne a été l'un des éléments clés de la profonde réforme de l'enseignement supérieur dans la République slovaque à partir de 1998, réforme qui visait à résoudre les problèmes de financement, de propriété, d'accès à l'enseignement supérieur et de qualité de l'enseignement. Le Processus de Bologne a été considéré comme un instrument de pré-adhésion à l'Union européenne: en impliquant l'enseignement supérieur slovaque dans cet espace européen en émergence, le pays tout entier devait gagner en reconnaissance.

14 Après l'approbation d'un document de cadrage en août 2000, puis l'intégration de la réforme dans le programme du gouvernement en 2002, une loi a été élaborée la même année pour mettre en œuvre les principes de Bologne, en particulier la mise en place progressive de trois niveaux d'études utilisant le système de crédits préconisé et le principe de l'accréditation des diplômes. Cette réforme renforçait la dimension internationale du système, grâce aux nouvelles possibilités de mobilité des étudiants et des enseignants slovaques à l'étranger. 
15 Pendant la dizaine d'années d'introduction du Processus de Bologne, l'enseignement supérieur a eu tendance à s'élargir et se diversifier, en même temps que les structures d'études se rapprochaient. En particulier, l'enseignement professionnel a été introduit au sein des universités traditionnelles pour associer connaissances générales et théoriques, recherche scientifique et activités créatives.

Mais les universités slovaques n'étaient pas bien préparées pour des changements aussi radicaux, et on peut en constater les conséquences aujourd'hui.

17 En effet, si les diplômes provenant des différents pays de l'Union européenne sont acceptés plus facilement et si la mise en place de programmes coordonnés entre des universités slovaques et étrangères est désormais possible, la législation reste assez confuse, les conditions du système d'accréditation sont encore très rigides et les acteurs acceptent difficilement une évolution des pratiques. Le nombre de programmes conjoints avec des universités étrangères augmente très lentement.

La mise en place des trois niveaux a été plus complexe que prévu, car il ne s'agissait pas d'intégrer un niveau intermédiaire (la licence, bakalar), mais bien de repenser le système avec un nouveau niveau de sortie pertinent et une qualification universitaire complète permettant de commencer une carrière professionnelle ou de choisir une spécialisation dans des études de master. Or dans de nombreux secteurs, l'adoption forcée de ce niveau de licence n'a pas respecté les demandes des spécialisations et les étudiants ont peu de débouchés sur le marché du travail.

L'adoption du système des crédits a accru la responsabilisation individuelle des étudiants. L'ouverture internationale du système d'enseignement supérieur slovaque a eu des conséquences positives, notamment pour la connaissance des langues étrangères ou l'identification européenne. Dans le même temps, les universités ont été amenées à s'ouvrir non seulement vers l'extérieur, mais aussi vers l'intérieur: la mobilité étudiante en Slovaquie même est un phénomène nouveau apparu après l'internationalisation.

20 La question de la qualité de l'enseignement reste au centre de nombreux débats en Slovaquie et a même constitué l'argument principal des premières manifestations étudiantes, en 2006. Les étudiants revendiquent toujours une augmentation de la qualité de l'enseignement, notamment le respect des maquettes des programmes accrédités, et dénoncent la hausse des frais de scolarité, tandis que les enseignants se plaignent surtout du niveau de salaire et de la complexification des procédures.

21 Pour certains, l'augmentation du nombre d'institutions d'enseignement supérieur a affaibli le prestige de l'enseignement supérieur dans l'opinion publique. La plupart des établissements n'admettent pas facilement la mesure de la qualité de l'enseignement à l'aide d'indicateurs quantitatifs (surtout dans le domaine scientifique), même si cette pratique s'impose de par la compétition européenne.

\section{Réformes et évolutions actuelles}

22 Le ministère slovaque en charge de l'enseignement supérieur et de la recherche actualise chaque année son plan à long terme. Les principaux objectifs définis en 2010 pour l'horizon 2014 sont de mieux prendre en compte les besoins des étudiants en termes de contenu et de méthodes; de favoriser les relations entre les employeurs potentiels et les diplômés; de créer un environnement académique stimulant (moins 
d'administration, approche partenariale de la formation, approche étudiant/client) ; de développer des "universités ouvertes" (otvorené univerzity) avec une meilleure information et la promotion de l'intérêt du public pour les activités des universités publiques; d'accroître le prestige du statut de professeur; de rendre l'enseignement supérieur plus accessible grâce à des aides sociales plus efficaces; de rendre l'environnement de l'enseignement supérieur plus stable avec, en particulier, un système nouveau de financements pluriannuels; d'analyser et prévoir les conditions de travail dans les universités.

Le Programme national de réforme (Národný program reforiem) défini dans le cadre de la stratégie de Lisbonne pour la période 2011-2013 puis le programme de stabilité pour la période 2012-2015 ont fait l'objet de recommandations de la part du Conseil de l'Union européenne et ont défini en 2011 un cadre de réforme ambitieux.

Le gouvernement avait envisagé, en particulier, de réformer les procédures d'accréditation (introduction d'une logique d'équipe pédagogique et non plus simplement d'un professeur garant unique pour un programme) ou encore de redéfinir les périmètres des formations par rapport à des champs d'études et non plus à des programmes normalisés, ce qui ouvre la possibilité de développer des projets de formation pluri ou interdisciplinaires, mieux adaptés. De plus, les critères d'obtention des grades académiques de professeur ou de maître de conférences habilité (docent) devraient être plus stricts et basés sur des standards internationaux (publications, recherches, activités internationales, etc.). Il était aussi envisagé de fusionner les trois catégories d'établissements en une seule (vysoké školy), car l'évaluation des différents établissements slovaques montre que les différences intra-université sont souvent plus importantes que les différences inter-universités. Il s'agissait de tenter d'améliorer le système à l'intérieur de chaque université et non plus de mettre en évidence des établissements d'excellence, qui en réalité comportent des composantes et des programmes de formation de qualité discutable. L'amélioration des études externes était une autre piste de réforme, notamment avec le changement de la durée de ces études (quatre ans pour le bakalar et trois pour le magister), afin d'en accroître la qualité. Enfin, des évolutions des modalités de financement étaient envisagées. La part du financement institutionnel réservé à la recherche devait être déterminée par le niveau international des résultats de recherche. Il s'agissait aussi d'accroître la transparence du système de financement institutionnel et des bourses pour la recherche et de favoriser une compétition entre structures de recherche publiques et privées pour l'obtention de financements.

Le ministre de l'Éducation, de la Science, de la Recherche et des Sports, Dušan Čaplovič, nommé le 4 avril 2012, reprend quelques-unes de ces pistes de réforme, mais envisage de revoir la conception du système d'enseignement supérieur slovaque sur des bases relativement différentes de celles du gouvernement précédent, dont les propositions étaient en partie d'inspiration néolibérale.

En premier lieu, le ministre considère que le nombre d'établissements d'enseignement supérieur est trop élevé par rapport à la taille et à la population du pays et que la qualité reste faible. Il a déclaré son intention de limiter le nombre d'établissements. Sept ou huit conserveraient le statut d'université de recherche, tandis que les autres se concentreraient sur les premier et, éventuellement, deuxième niveaux d'études, un objectif réaliste si l'on considère comme nécessaire la fusion de certains petits établissements voisins ${ }^{2}$. Le ministre entend également limiter le nombre de 
«professeurs volants» (lietajúcich profesorov), afin d'obtenir une implication plus forte des universitaires ${ }^{3}$, mais il n'est pas certain que cela augmentera la qualité des programmes, car ces cumuls visent surtout à compenser la faiblesse des salaires des universitaires, une situation qu'il faudrait prendre en compte.

Le ministre prévoit le maintien du budget de l'enseignement supérieur (environ 400 millions sur 16,9 milliards d'euros en 2011), mais envisage de le répartir de manière plus efficace, en particulier en limitant les inscriptions dans certains domaines d'études. Sont ainsi cités l'économie, le droit, les sciences sociales et les sciences humaines, qui fournissent un nombre élevé de diplômés chômeurs.

Néanmoins, les recommandations du Conseil de l'Union européenne, qui avaient dicté les idées de réforme du précédent gouvernement, sont toujours d'actualité : il s'agit de poursuivre les efforts visant à améliorer la qualité de l'enseignement supérieur, à adapter l'enseignement supérieur à la demande du marché et à inciter les personnes peu qualifiées à étudier et se former tout au long de la vie.

La Slovaquie est un exemple de «bon élève » du Processus de Bologne, mis en place à marche forcée mais dans un contexte très favorable d'intégration européenne et d'europhilie massive de la population et, donc, avec l'adhésion des acteurs. L'autonomie relative des établissements, majoritairement publics, bute désormais sur une volonté politique, assez pragmatique, de réguler les flux et les spécialités en fonction du marché du travail et d'optimiser les dépenses publiques. Ainsi, le gouvernement reprend un contrôle sur les universités qui avait été relativement distendu. La faiblesse relative des salaires du personnel universitaire, liée à la contrainte budgétaire et à l'orthodoxie de la politique gouvernementale de ce pays, qui a introduit l'euro en 2009, ne constitue pas une incitation au départ vers l'Ouest, comme cela se produit, par exemple, en Bulgarie : les universités slovaques, leurs universitaires et leurs étudiants se sont « européanisés » et l'ampleur des mobilités, plus ou moins longues, explique en -partie une intégration en fin de compte réussie. 嘕

\section{BIBLIOGRAPHIE}

CÔME Thierry \& MESKOVA Ludmila (2011) : Les Universités en Europe centrale, 20 ans après, économie et management, Bruxelles, Bruylant.

JENSEN Henrik Toft, KRALJ Alojz, McQUILLAN Don \& REICHERT Sybille (2008): The Slovak Higher Education System and its Research Capacity, EUA Sectoral Report, European University Association (EUA), Institutional Evaluation Programme.

KOSOVÁ Béata \& PORUBSKÝ Štefan (2007): « Educational transformation in Slovakia: The ongoing search for a solution $»$, Orbis Scholae, an $1, n^{\circ} 2$.

ROUET Gilles \& ROŠTEKOVÁ Mária (2013) : Regards croisés sur la Slovaquie contemporaine, Presses universitaires de Banska Bystrica.

Slovaquie, Ministère de l'éducation, Higher education in Slovakia. From Bologna towards the European Higher Education Area, Bratislava, 2004. 


\section{NOTES}

1. Université slovaque de la santé de Bratislava, -Académie militaire et Académie de police. Les établissements d'enseignement supérieur publics sont les universités traditionnelles, les universités techniques, l'université d'économie, l'université vétérinaire et l'établissement d'enseignement supérieur d'art.

2. Par exemple, Trnava, ville de 66000 habitants, compte deux universités et une filiale de l'université technique de Bratislava.

3. Il s'agit de limiter à 58 heures par semaine le temps de travail autorisé pour un enseignant, donc de ne plus autoriser $20 \%$ des universitaires à avoir deux emplois à temps plein (37,5 heures par semaine par emploi).

\section{INDEX}

Index géographique : Slovaquie

Mots-clés : autonomie, budget de l'éducation, enseignement supérieur, programme d'enseignement, qualité de l'enseignement, réforme, système d'enseignement, université, processus de Bologne, assurance qualité, curriculum, LMD : Licence-Master-Doctorat

\section{AUTEURS}

\section{BEATA KOSOVÁ}

Professeur de sciences pédagogiques, rectrice de l'université Matej Bel de Banska Bystrica depuis 2007, présidente de la commission pédagogique au sein de la conférence des recteurs de Slovaquie.

\section{GILLES ROUET}

Professeur de sciences de l'éducation, membre du GEPECS, université Paris Descartes, chaire Jean Monnet ad personam en études interdisciplinaires sur l'Union européenne, et attaché de coopération universitaire et scientifique en Slovaquie (2004-2008) et en Bulgarie (depuis décembre 2010). 